\title{
Inappropriate sinus tachycardia in post-COVID-19 syndrome
}

Júlia Aranyó ${ }^{1}$, Victor Bazan ${ }^{1}$, Gemma Lladós ${ }^{2}$, Maria Jesús Dominguez ${ }^{3}$, Felipe Bisbal ${ }^{1}$,
Marta Massanella ${ }^{4}$, Axel Sarrias ${ }^{1}$, Raquel Adeliño ${ }^{1}$, Ariadna Riverola ${ }^{1}$, Roger Paredes ${ }^{4}$,
Bonaventura Clotet ${ }^{2,4}$, Antoni Bayés-Genís ${ }^{1,5,6}$, Lourdes Mateu ${ }^{2,6,7,8}$ \& Roger Villuendas $s^{1,5,6,8}$ R

Inappropriate sinus tachycardia (IST) is a common observation in patients with post-COVID-19 syndrome (PCS) but has not yet been fully described to date. To investigate the prevalence and the mechanisms underlying IST in a prospective population of PCS patients. Consecutive patients admitted to the PCS Unit between June and December 2020 with a resting sinus rhythm rate $\geq 100 \mathrm{bpm}$ were prospectively enrolled in this study and further examined by an orthostatic test, 2D echocardiography, 24-h ECG monitoring (heart rate variability was a surrogate for cardiac autonomic activity), quality-of-life and exercise capacity testing, and blood sampling. To assess cardiac autonomic function, a 2:1:1 comparative sub-analysis was conducted against both fully recovered patients with previous SARS-CoV-2 infection and individuals without prior SARS-CoV-2 infection. Among 200 PCS patients, 40 (20\%) fulfilled the diagnostic criteria for IST (average age of $40.1 \pm 10$ years, $85 \%$ women, $83 \%$ mild COVID-19). No underlying structural heart disease, proinflammatory state, myocyte injury, or hypoxia were identified. IST was accompanied by a decrease in most heart rate variability parameters, especially those related to cardiovagal tone: pNN50 (cases $3.2 \pm 3$ vs. recovered $10.5 \pm 8$ vs. non-infected $17.3 \pm 10 ; p<0.001)$ and HF band $(246 \pm 179$ vs. $463 \pm 295$ vs. $1048 \pm 570$, respectively; $p<0.001$ ). IST is prevalent condition among PCS patients. Cardiac autonomic nervous system imbalance with decreased parasympathetic activity may explain this phenomenon.

More than 100 million people have been infected with SARS-CoV-2 worldwide. Most of these patients experience mild symptoms that do not warrant hospital admission. However, approximately $20-40 \%$ of patients remain symptomatic weeks, or even months, after overcoming the acute infection phase ${ }^{1}$. This phenomenon is regarded as ongoing symptomatic COVID-19 or post-COVID-19 syndrome (PCS) when remnant symptoms persist from 4 to 12 weeks and for more than 12 weeks, respectively ${ }^{2}$. Clinical manifestations of PCS usually include fatigue, chest pain, joint/muscle pain, dizziness, fever, shortness of breath, gastrointestinal symptoms, headache, sore throat, neurocognitive disorder, and altered sleep structure. Limited understanding of the pathological mechanisms underlying PCS represents a critical challenge to effectively testing and treating this syndrome. Injury to the autonomic nervous system (ANS) has recently been suggested to be responsible for many of the aforementioned manifestations and may be key in the pathogenesis of PCS 3 .

At the cardiovascular level, ANS dysfunction produces orthostatic syndromes, such as orthostatic hypotension and postural orthostatic tachycardia syndrome (POTS), chest pain, and cardiac arrhythmias, including inappropriate sinus tachycardia (IST) ${ }^{4}$. Postural orthostatic tachycardia has already been described in the setting of PCS ${ }^{3,5}$. In our initial experience with PCS patients, IST, which often overlaps with POTS, is also a common observation that has not been fully described to date. Similar to POTS, decreased parasympathetic activity has been postulated in the etio-pathogenesis of IST ${ }^{6,7}$. The aim of this study was to investigate the prevalence and underlying pathophysiological mechanisms of IST in a consecutive and prospective population of PCS patients.

\footnotetext{
${ }^{1}$ Cardiology Department, Heart institute, Hospital Universitari Germans Trias I Pujol, Carretera de Canyet s/n, 08916 Badalona, Spain. ${ }^{2}$ Department of Infectious Disease, Hospital Universitari Germans Trias I Pujol, Badalona, Spain. ${ }^{3}$ Emergency Department, Hospital Universitari Germans Trias I Pujol, Badalona, Spain. ${ }^{4}$ AIDS Research Institute (IrsiCaixa), Badalona, Spain. ${ }^{5}$ Centro de Investigación Biomédica en Red Enfermedades Cardiovascualres (CIBERCV), Madrid, Spain. ${ }^{6}$ Autonomous University of Barcelona, Barcelona, Spain. ${ }^{7}$ Centro de Investigación Biomédica en Red Enfermedades Respiratorias (CIBERES), Madrid, Spain. ${ }^{8}$ These authors contributed equally: Lourdes Mateu and Roger Villuendas. ${ }^{\square}$ email: rogervilluendas@hotmail.com
} 


\section{Methods}

Study population. At our institution, patients with persistent symptoms, such as tiredness, shortness of breath, dizziness, brain fog, chest pain, or headache, 3 months after an acute SARS-CoV-2 infection are referred to a multi-disciplinary PCS unit supported by infectologists, cardiologists, neurologists, rheumatologists, nutritionists, rehabilitators, and psychologists. All consecutive patients seen at this unit from June to December 2020 underwent a resting 12-lead ECG. Patients with sinus rhythm rates $\geq 100 \mathrm{bpm}$ were prospectively enrolled in the study database and underwent further cardiovascular assessment. The assessment included an orthostatic test during a 10-min period of standing (to detect concomitant POTS), 2-D echocardiography, 24-h Holter monitoring, a quality-of-life test (EQ-5D-5L), 6-min walking test (6MWT), and blood sample collection to the search for biological markers of inflammation and myocardial damage. The EQ-5D-5L has five response levels: no problems (level 1), slight, moderate, severe, and extreme problems (level 5).

Secondary causes of tachycardia, such as anemia, thyroid pathology, pregnancy, infection, or pulmonary embolism, were investigated, and patients with a systemic condition justifying tachycardia were excluded from the study analysis. Patients using sympathomimetic drugs were also excluded.

Study approval. The study was approved by the institutional ethics committee (Hospital Universitari Germans Trias i Pujol, Badalona, Barcelona, Spain; PI 20-288). The participants signed a written informed consent form before enrolling in the study. All research activities were carried out in accordance with the Declaration of Helsinki.

Definition of inappropriate sinus tachycardia. Following conventional criteria, IST was defined as a symptomatic sinus rhythm rate $\geq 100 \mathrm{bpm}$ at rest with a mean 24 -h heart rate above $90 \mathrm{beats} / \mathrm{min}$ in the absence of any acute physiological demand or conditions known to commonly produce sinus tachycardia ${ }^{8}$.

Assessment of cardiac autonomic function. In this study, we based our assessment of ANS imbalance on the time-and-frequency-domain heart rate variability (HRV) parameters obtained during 24-h ECG monitoring. Time-domain measurements included the average RR interval (in $\mathrm{ms}$ ), the standard deviation of the inter-beat interval (SDNN, in $\mathrm{ms}$ ), and the percentage of adjacent NN intervals that differed from each other by more than $50 \mathrm{~ms}$ (PNN50, \%). Frequency-domain parameters included the very low frequency (VLF; $0.003-0.04 \mathrm{~Hz}$ ), low frequency (LF; 0.04-0.15 Hz), and high frequency (HF; $0.15-0.40 \mathrm{~Hz}$ ) bands. The ratio between the LF and HF bands was also calculated. Briefly, the HF and PNN50 are regarded as specific indicators of the parasympathetic influence on the heart rate, whereas the LF and VLF components have a complex physiology that integrates both the sympathetic and parasympathetic components ${ }^{9}$. The remaining parameters are less specific to a determined sympathetic versus parasympathetic influence on the heart rate and, thus, become less useful in characterizing a specific ANS disturbance.

In the absence of reliable reference values for the HRV parameters in the literature, we conducted a 2:1:1 comparative sub-study using two healthy populations. The sub-study included the following groups: group 1, all IST patients (cases); group 2, age- and gender-matched PCR-confirmed SARS-COV-2 patients without IST criteria; and group 3, age- and gender-matched patients who had no history of SARS-COV-2 disease, as confirmed by negative serology. Patients in group 2 were also matched by disease chronology, and their acute infection had to have the same severity and be within the same 1-month period as the corresponding cases. The severity of the infection was determined by the following criteria. Mild disease was defined as the presence of symptoms without evidence of viral pneumonia or hypoxia; moderate disease as hospitalization due to abnormal chest $\mathrm{X}$-ray, hypoxia, or sepsis; and critical disease as requiring intensive care management. This 2:1:1 comparative design allowed us to establish study reference values for the assessment of HRV and to characterize presumable damage to the sympathetic versus parasympathetic input to the heart rate in the setting of PCS.

All of the Holter recordings were analyzed using an AFT $1000+$ B recorder (Holter Supplies SAS, Paris, France).

Statistical analysis. Demographic data were summarized by basic descriptive statistics in the three groups. All analyses treated the three groups independently, whereas the matching process for every two "cases" was individual. For qualitative variables, numbers and percentages within specified groups were calculated, and $p$ values were obtained using $\chi^{2}$ tests. For quantitative variables, the arithmetic mean and standard deviation (SD) or median and interquartile range were reported as appropriate. Continuous variables were tested for normal distribution using Q-Q plots. The quantitative variables were compared between the three groups using a oneway ANOVA model and $p$-values for post-hoc comparisons were adjusted using the Scheffe method. All statistical analyses were performed using SPSS version 25.0 (IBM, Armonk, NY, USA). Significance was set at $p<0.05$.

\section{Results}

During the study period, 200 patients visited the PCS unit due to persistent symptoms beyond the third month of acute infection. Forty-four patients with a resting heart rate $\geq 100 \mathrm{bpm}$ were initially screened, 4 of whom were excluded due to mean 24 -h heart rate $<90 \mathrm{bpm}(\mathrm{n}=2)$, hyperthyroidism $(\mathrm{n}=1)$, or severe mitral regurgitation $(n=1)$. Therefore, 40 patients fulfilled the strict diagnostic criteria for IST, yielding an estimated prevalence of the disorder of $20 \%$. No patient had complained of palpitations prior to the SARS-CoV-2 infection, endorsing the principle of post-infective IST. No patient was under any cardiovascular treatment at the time of the evaluation. The IST subjects had a mean heart rate of $105 \pm 2 \mathrm{bpm}$ supine and $125 \pm 11 \mathrm{bpm}$ in the upright position. The baseline characteristics of the 40 IST cases and their matched controls are presented in Table 1 . All patients 


\begin{tabular}{|c|c|c|c|c|}
\hline & $\begin{array}{l}\text { Inappropriate sinus tachycardia } \\
\text { (Group 1) } \\
\mathrm{N}=40\end{array}$ & $\begin{array}{l}\text { Fully recovered (Group 2) } \\
\mathrm{N}=19\end{array}$ & $\begin{array}{l}\text { Uninfected (Group 3) } \\
\mathrm{N}=17\end{array}$ & $P$ value \\
\hline \multicolumn{5}{|c|}{ Demographic and clinical characteristics } \\
\hline Age (years) & $40.1 \pm 10$ & $42.2 \pm 11$ & $39.5 \pm 13$ & 0.243 \\
\hline Males, n (\%) & $6(15)$ & $2(11)$ & $2(12)$ & 0.655 \\
\hline Body mass index, mean \pm SD & $25.2 \pm 6.1$ & $24.5 \pm 3.6$ & $22.5 \pm 2.3$ & 0.374 \\
\hline Smoking, n (\%) & $1(3)$ & $1(5)$ & 0 & 0.623 \\
\hline Hypertension, n (\%) & $3(8)$ & 0 & 0 & 0.589 \\
\hline Hyperlipidemia, n (\%) & $3(8)$ & 0 & 0 & 0.589 \\
\hline Diabetes mellitus, n (\%) & 0 & 0 & 0 & NA \\
\hline Asthma, n (\%) & $4(10)$ & $1(5)$ & 0 & 0.734 \\
\hline Environmental allergy, n (\%) & $10(25)$ & 0 & 0 & 0.01 \\
\hline \multicolumn{5}{|l|}{\begin{tabular}{|l|} 
Symptoms of the acute infection \\
\end{tabular}} \\
\hline Palpitations, n (\%) & $36(90)$ & $1(5)$ & - & $<0.001$ \\
\hline Dyspnea, n (\%) & $33(83)$ & $3(16)$ & - & $<0.001$ \\
\hline Myalgia and joint pain, $\mathrm{n}(\%)$ & $32(80)$ & $17(89)$ & - & 0.545 \\
\hline Chest pain, $\mathrm{n}(\%)$ & $31(78)$ & $4(21)$ & - & $<0.001$ \\
\hline Fever, n (\%) & $29(73)$ & $16(84)$ & - & 0.462 \\
\hline Headache, n (\%) & $29(73)$ & $7(37)$ & - & 0.007 \\
\hline Dizziness, n (\%) & $21(53)$ & $1(5)$ & - & 0.002 \\
\hline Diarrhea, n (\%) & $21(53)$ & $3(16)$ & - & 0.003 \\
\hline Anosmia, n (\%) & $19(48)$ & $15(79)$ & - & 0.03 \\
\hline Ageusia, n (\%) & $19(48)$ & $8(42)$ & - & 0.454 \\
\hline Dermatologic alterations, $\mathrm{n}(\%)$ & $14(35)$ & $1(5)$ & - & 0.009 \\
\hline \multicolumn{5}{|l|}{\begin{tabular}{|l|} 
Severity of clinical presentation \\
\end{tabular}} \\
\hline Mild & $33(83)$ & $16(84)$ & - & 0.387 \\
\hline Moderate & $6(15)$ & $3(16)$ & - & 0.550 \\
\hline Intensive care management & $1(3)$ & 0 & - & 0.254 \\
\hline
\end{tabular}

Table 1. Demographic and clinical characteristics of the cases and their matched controls. Values are expressed as mean \pm standard deviation unless otherwise stated. A P value of $<0.05$ is considered statistically significant. Significant values are in [bold].

were Caucasian. Thirty-four (85\%) were women, with a mean age of $40.1 \pm 10$ years. All patients had normal 2-D echocardiography results, and no remnant respiratory disease was identified in any patient. All patients had $\mathrm{O}_{2}$ saturation $>97 \%$. Notably, IST patients had a higher prevalence of environmental allergy compared to the control group ( $25 \%$ vs. $0 \%$; $p=0.01)$.

During the acute phase of SARS-CoV-2 infection, 33 patients (83\%) had experienced mild symptoms not requiring hospital admission; 6 patients (15\%) had moderate disease with pulmonary infiltrates and required hospitalization; and only 1 patient (3\%) required intensive care management. Compared to fully recovered patients, patients with PCS and IST more frequently complained of palpitations ( $90 \%$ vs. $5 \% ; p<0.001)$, dyspnea ( $82 \%$ vs. $16 \% ; p<0.001)$, chest pain $(78 \%$ vs. $21 \% ; p<0.001)$, headache $(73 \%$ vs. $37 \% ; p=0.007)$, dizziness $(53 \%$ vs. $5 \% ; p=0.002)$, diarrhea ( $53 \%$ vs. $16 \% ; p=0.003)$, and dermatological alterations (35\% vs. $5 \%$; $p=0.009)$. Interestingly, IST patients had a lower incidence of anosmia ( $48 \%$ vs. $79 \% ; p=0.03$ ).

The results of the exercise capacity and quality of life assessment are presented in Table 2, along with the results of the laboratory tests. Echocardiography yielded normal results in all patients. Overall, biochemistry data were consistent with a lack of inflammation or myocardial damage at this stage after the acute phase of SARSCoV-2 infection. The 6MWT showed that IST patients had a significantly diminished exercise capacity, with a median walking distance of $392 \pm 83 \mathrm{~m}$, which is only $60 \%$ of the estimated reference distance after adjusting for age, sex, and body mass index. Impaired quality of life was also identified, as suggested by a mean score in the health-state scale of 39 out of 100 points. The most affected domains were mobility (mean score 3.6), usual activities (mean score 3.5), and pain/discomfort (mean score 3 ).

Results of the 24-h ECG monitoring are summarized in Table 3 and Fig. 1. Patients with IST had a higher mean heart rate, predominantly during the daytime, compared to recovered asymptomatic and uninfected subjects ( $98 \pm 6$ vs. $84 \pm 8$ vs. $81 \pm 6$ bpm, respectively; $p<0.001$ ). The burden of supraventricular premature beats was lower in IST-PCS patients. No differences were observed in the maximum and minimum heart rates. All HRV variables were significantly diminished among patients with IST compared to both the recovered subjects and the uninfected group, with a significant decrease in the following time-domain parameters: daytime pNN50 (3.2 \pm 3 vs. $10.5 \pm 8$ vs. $17.3 \pm 10.0$, respectively; $p<0.001)$ and daytime $\operatorname{SDNN}(95.0 \pm 25$ vs. $121.5 \pm 34$ vs. $138.1 \pm 25$, respectively; $p<0.001)$. A significant decrease in frequency-domain parameters was also observed in PCS patients with IST: VLF $(1463.1 \pm 538$ vs. $2415.7 \pm 1361$ vs. $3931 \pm 2194$, respectively; $p<0.001)$, LF $(670.2 \pm 380$ vs. $1093.2 \pm 878$ vs. $1801.5 \pm 800$, respectively; $p<0.001)$, and $H F(246.0 \pm 179$ vs. $463.7 \pm 295$ vs. $1048.5 \pm 570$, 


\begin{tabular}{|c|c|c|}
\hline & Reference values & Mean values \\
\hline \multicolumn{3}{|l|}{ Laboratory tests } \\
\hline NT-proBNP (pg/mL) & $<125$ & $67.6 \pm 59.6$ \\
\hline Hs-Troponin I (pg/mL) & $<14$ & $3.09 \pm 4.2$ \\
\hline Leucocytes $\left(\times 10^{9} / \mathrm{L}\right)$ & $4.00-11.00$ & $6.83 \pm 1.7$ \\
\hline Hemoglobin $(\mathrm{g} / \mathrm{dL})$ & $12.0-16.0$ & $13.4 \pm 0.7$ \\
\hline $\mathrm{TSH}(\mu \mathrm{m} / \mathrm{U})$ & $0.350-4.940$ & $1.4 \pm 0.5$ \\
\hline IL-6 (pg/mL) & $0-6.40$ & $2.2 \pm 1.1$ \\
\hline Ferritin $(\mathrm{ng} / \mathrm{mL})$ & $15-160$ & $50.4 \pm 37.4$ \\
\hline C-reactive protein $(\mathrm{mg} / \mathrm{L})$ & $0.00-5.00$ & $1.1 \pm 1.3$ \\
\hline D-Dimer (ng/mL) & $0-500$ & $247.7 \pm 133.2$ \\
\hline Fibrinogen & $150-450$ & $374.9 \pm 54.1$ \\
\hline 24-h urine 3-Metoxiadrenaline (mg/L) & $0.02-0.350$ & $0.123 \pm 0.120$ \\
\hline 24-h urine 3-Metoxinoradrenaline (mg/L) & $0.03-0.440$ & $0.178 \pm 0.189$ \\
\hline \multicolumn{3}{|l|}{ Exercise capacity test } \\
\hline 6MWT (m) & & $392.7 \pm 83.2$ \\
\hline 6MWT theoretical (\%) & & $60.1 \pm 12.1$ \\
\hline Quality of life test (EQ-5D-5L) & Score reference values ${ }^{\mathrm{a}}$ & Mean values \\
\hline Mobility & $1-5$ & $3.6 \pm 1.1$ \\
\hline Self-care & $1-5$ & $2.4 \pm 1.2$ \\
\hline Usual activities & $1-5$ & $3.5 \pm 0.9$ \\
\hline Pain/discomfort & $1-5$ & $3.0 \pm 1.0$ \\
\hline Anxiety/depression & $1-5$ & $2.9 \pm 1.2$ \\
\hline Health state scale (Visual Analogue Scale) & $0-100$ & $39.6 \pm 20.7$ \\
\hline
\end{tabular}

Table 2. Characterization of patients with IST. Values are expressed as mean \pm standard deviation unless otherwise stated. NT-proBNP N-terminal pro B-type natriuretic peptide, TSH thyroid-stimulating hormone,


each health dimension: 1: No problems, 2: Slight problems, 3: Moderate problems, 4: Severe problems, 5: Unable/Extreme. For Health state scale, 0 means the best health you can imagine, 100 means the worst health you can imagine.

respectively; $p<0.001)$. Numerical but non-significant differences were also observed between both control groups, with the fully recovered patients presenting with higher heart rates and lower HRV than the uninfected subjects.

An illustrative example of 24-h ECG monitoring showing altered versus normal HRV in a PCS patient vs. control is shown in Fig. 2.

\section{Discussion}

To the best of our knowledge, this is the first prospective series of consecutive PCS patients in whom a comprehensive cardiovascular evaluation has been performed for the investigation of IST. We found that IST is prevalent among PCS patients (affecting 20\% in our series), and this disorder was more common in young women without previous comorbidities and with mild SARS-CoV-2 infection. IST provides a plausible explanation for some of the prevalent symptoms of fatigue, impaired exercise capacity, and palpitations that characterize PCS and limit the affected individuals' ability to carry out a normal life (Fig. 3). Finally, our results suggest a major role of the ANS in the pathophysiology of IST. This is supported by the 24-h ECG monitoring, as IST was accompanied by a decrease in most HRV parameters, predominantly during the daytime, and the most reduced components were those related to the cardiovagal tone (pNN50 and HF band). Accordingly, the loss of HRV is suggestive of a cardiac ANS imbalance with decreased parasympathetic activity and compensatory sympathetic activation. Hypoactivity of the parasympathetic tone could explain not only our findings of PCS-related IST, but also other prevalent symptoms in this setting, such as fatigue, gastrointestinal discomfort, headache, sore throat, neurocognitive disorder, and altered sleep structure (Central Illustration). Our findings are consistent with previous investigations suggesting that PCS could be a form of post-infectious dysautonomia.

Anxiety disorders, an acknowledged cause of sinus tachycardia, were not systematically evaluated in our patient population. However, the observed low HRV in our cohort and manifest physical limitations during the 6MWT makes anxiety-driven IST rather unlikely.

The mechanisms of IST, with or without previous viral infection, are poorly understood and investigated, but many of the postulated mechanisms include alterations in the nervous system: sympathovagal imbalance, beta-adrenergic receptor hypersensitivity, and brain stem dysregulation, among others. Considering this, it seems reasonable that the mechanisms leading to IST after SARS-CoV-2 infection are mixed, with injury of the ANS, which constantly regulates heart rate and vascular tone, playing an important role. 


\begin{tabular}{|c|c|c|c|c|c|c|}
\hline & \multirow{2}{*}{$\begin{array}{l}\text { Inappropriate } \\
\text { sinus tachycardia } \\
\mathrm{N}=40\end{array}$} & \multirow[b]{2}{*}{$\begin{array}{l}\text { Fully recovered } \\
\mathrm{N}=19\end{array}$} & \multirow[b]{2}{*}{$\begin{array}{l}\text { Uninfected } \\
\mathrm{N}=17\end{array}$} & \multicolumn{3}{|l|}{$P$ value } \\
\hline & & & & IST vs recovered & IST vs uninfected & $\begin{array}{l}\text { Recovered vs } \\
\text { uninfected }\end{array}$ \\
\hline \multicolumn{7}{|c|}{\begin{tabular}{|l} 
Time-domain parameters \\
\end{tabular}} \\
\hline Mean HR (bpm) & $93.6 \pm 3$ & $78.7 \pm 7$ & $74.3 \pm 5$ & $<0.001$ & $<0.001$ & 0.309 \\
\hline $\begin{array}{l}\text { Maximum HR } \\
\text { (bpm) }\end{array}$ & $154.6 \pm 16$ & $148.9 \pm 24$ & $140.8 \pm 18$ & 0.653 & 0.167 & 0.657 \\
\hline $\begin{array}{l}\text { Minimum HR } \\
\text { (bpm) }\end{array}$ & $59 \pm 10$ & $53.8 \pm 9$ & $51.9 \pm 4$ & 0.373 & 0.177 & 0.845 \\
\hline $\begin{array}{l}\text { Supraventricular } \\
\text { PB }\end{array}$ & $191.6 \pm 313$ & $546.6 \pm 665$ & $955.8 \pm 1180$ & 0.172 & 0.003 & 0.234 \\
\hline Ventricular PB & $85.6 \pm 120$ & $354.1 \pm 542$ & $186.9 \pm 287$ & 0.018 & 0.628 & 0.362 \\
\hline $\begin{array}{l}\text { Mean daytime HR } \\
(\mathrm{bpm})\end{array}$ & $97.5 \pm 6$ & $84.1 \pm 8$ & $80.6 \pm 6$ & $<0.001$ & $<0.001$ & 0.656 \\
\hline $\begin{array}{l}\text { Daytime PNN50 } \\
(\%)\end{array}$ & $3.2 \pm 3$ & $10.5 \pm 8$ & $17.3 \pm 10$ & 0.001 & $<0.001$ & 0.019 \\
\hline Daytime SD (ms) & $95.0 \pm 25$ & $121.5 \pm 34$ & $138.1 \pm 25$ & 0.011 & $<0.001$ & 0.270 \\
\hline $\begin{array}{l}\text { Mean nighttime } \\
\text { HR (bpm) }\end{array}$ & $80.0 \pm 7$ & $71.2 \pm 7$ & $67.4 \pm 5$ & 0.001 & $<0.001$ & 0.405 \\
\hline \begin{tabular}{|l|}
$\begin{array}{l}\text { Nighttime PNN50 } \\
(\%)\end{array}$ \\
\end{tabular} & $8.4 \pm 8$ & $16.6 \pm 15$ & $21.4 \pm 11$ & 0.051 & 0.004 & 0.498 \\
\hline Nighttime SD (ms) & $101.3 \pm 28$ & $144.5 \pm 42$ & $145.4 \pm 39$ & $<0.001$ & 0.003 & 0.997 \\
\hline \multicolumn{7}{|c|}{ Spectral-domain parameters } \\
\hline VLF (Hz) & $1463.1 \pm 538$ & $2415.7 \pm 1361$ & $3931.1 \pm 2194$ & 0.044 & $<0.001$ & 0.007 \\
\hline LF (Hz) & $670.2 \pm 380$ & $1093.2 \pm 878$ & $1801.5 \pm 800$ & 0.092 & $<0.001$ & 0.015 \\
\hline $\mathrm{HF}(\mathrm{Hz})$ & $246.0 \pm 179$ & $463.7 \pm 295$ & $1048.5 \pm 570$ & 0.060 & $<0.001$ & $<0.001$ \\
\hline LF/HF ratio $(\mathrm{Hz})$ & $3.6 \pm 1$ & $2.7 \pm 1.3$ & $2.0 \pm 1$ & 0.259 & 0.040 & 0.612 \\
\hline
\end{tabular}

Table 3. 24-h ECG monitoring and HRV parameters. Values are presented in mean \pm standard deviation. $H R$ heart rate, $S D$ standard deviation of the interbeat interval, $P N N 50$ percentage of adjacent NN intervals that differ from each other by more than $50 \mathrm{~ms}$, $V L F$ very low frequency, $L F$ low frequency, $H F$ high frequency. Significant values are in [bold].

Some studies have shown that COVID-19 has significant cardiovascular involvement, but no previous research has focused on IST after SARS-CoV-2 infection. However, this is not the first time that IST has been described after coronavirus infection. In 2006, Yu et al. found that IST was the most common cardiovascular complication in a cohort of 121 patients with SARS. IST occurred in the absence of fever and was persistent in nearly $40 \%$ of patients during 3 weeks of follow-up. The authors observed that cardiovascular outcomes did not correlate with the occurrence of hypoxemia, admission to the intensive care unit, or analytical abnormalities ${ }^{9}$. Madjid et al. previously described a weak association between acute Severe Acute Respiratory Syndrome Coronavirus-1 (SARS-CoV-1) and acute Middle Respiratory Syndrome (MERS) and cardiovascular complications, such as arrhythmia and transient diastolic dysfunction. In addition, a review of 28 studies evaluating the long-term manifestations of SARS-CoV-1 and MERS observed that the most common symptoms were fatigue, dyspnea, and weakness, similar to PCS ${ }^{10}$.

On the one hand, post-infectious dysautonomia has previously been described in relation to other pathogens, including Chagas disease, human immunodeficiency virus (HIV), Epstein-Barr virus, and rabies virus ${ }^{11,12}$. Autonomic dysfunction is relatively common among HIV-infected patients, as inferred from a decrease in the HRV in the early stages of infection in many of these patients ${ }^{13}$. On the other hand, that patients with IST or POTS often report experiencing a previous trigger, such as a viral infection ${ }^{14,15}$. In previous observational studies, previous infectious illness was the precipitating event for IST in 5-10\% of cases, and the reported pathogens were the influenza virus, Epstein-Barr virus, and herpes zoster, among others ${ }^{16}$. All of these studies mentioned ANS disruption. A number of mechanisms have been proposed to explain the occurrence of ANS dysfunction after a viral infection: denervation of the ANS, virus-dependent tissue damage due to persistent infection, and immune-mediated injury, among others. However, the prevalence and the mechanisms underlying the cardiovascular consequences of post-infectious dysautonomia are not clear and need to be investigated further.

As with other pathogens, there is convincing evidence that SARS-CoV-2 can damage the ANS. Previous studies have suggested a number of concurrent mechanisms, including direct brain invasion across the ethmoid bone or via the olfactory bulb during acute infection or blood dissemination of the virus and use of the ACE2 receptor for intracellular penetration. This receptor is also present on the glial cells and neurons. Several lines of evidence also support "indirect" mechanisms as the most important mechanisms involved in neurological injury, including vasculitis, thrombosis, and endothelial damage, along with exaggerated inflammation and immune responses ${ }^{17-22}$.

Furthermore, the evidence not only supports that SARS-CoV-2 can affect the nervous system during the acute phase, there is growing evidence in patients with orthostatic syndromes and syncope following SARS-CoV-2 infection that endorses a patho-physiological link between PCS and ANS dysfunction. A clear example of the 

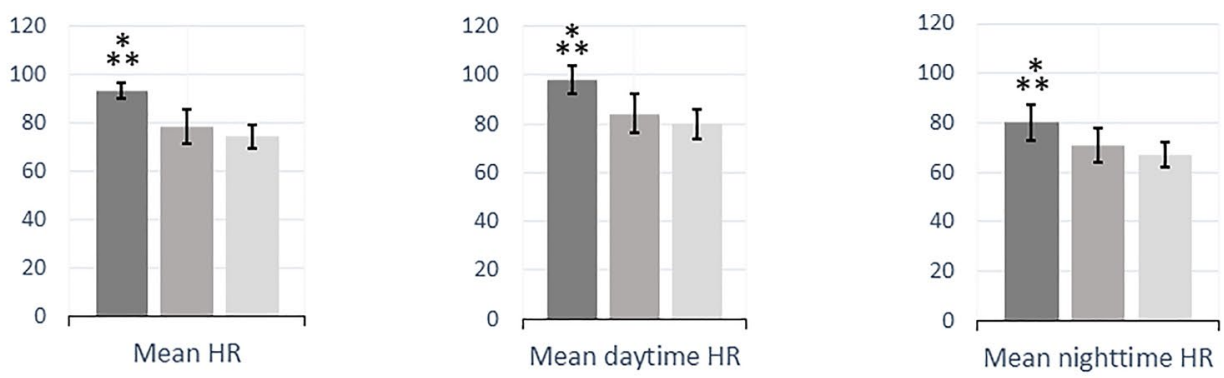

IST

$\square$ Fully recovered

$\square$ Uninfected
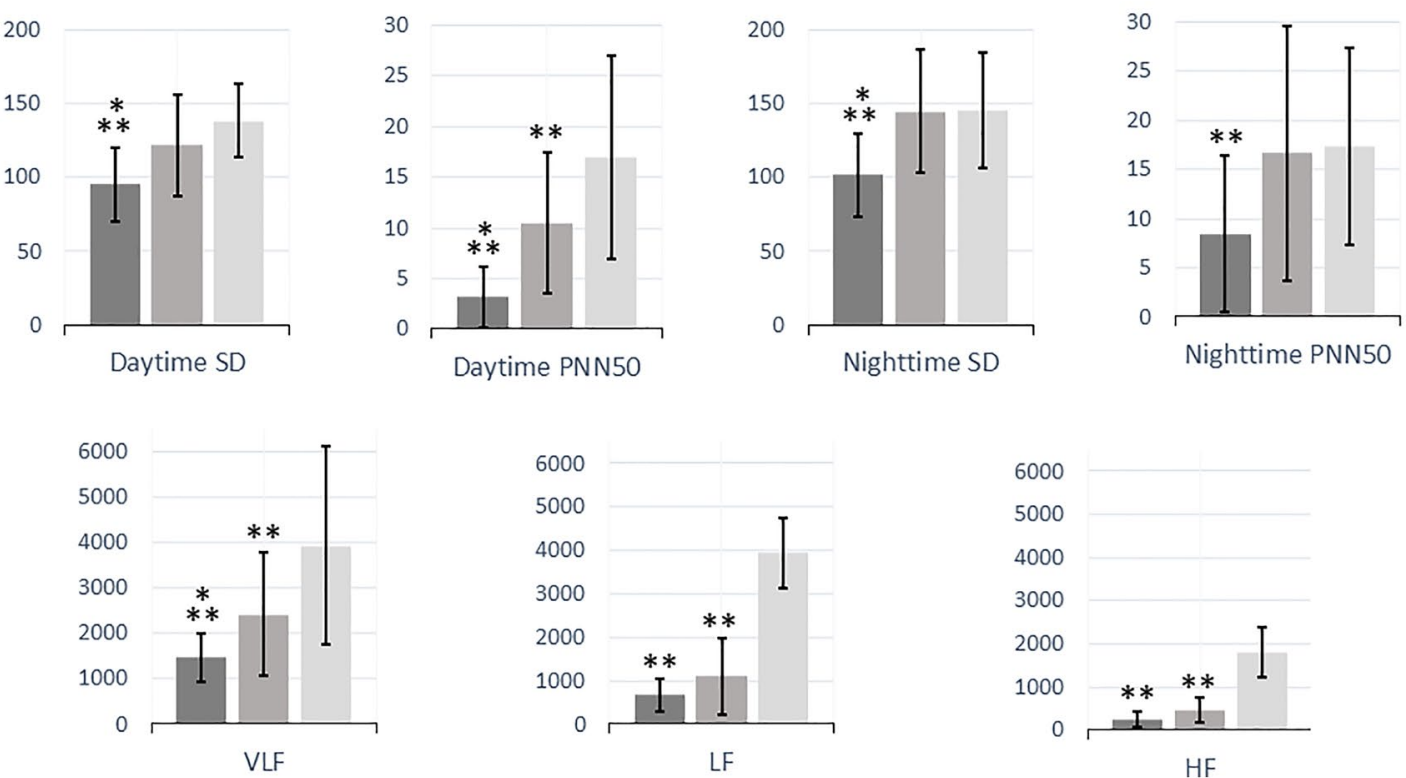

Figure 1. 24-h ECG monitoring and HRV parameters. HRV parameters in the three studied groups: IST, fully recovered and uninfected subjects. HR indicates heart rate; PNN50, percentage of adjacent NN intervals that differ from each other by more than $50 \mathrm{~ms}$; SD, standard deviation of the interbeat interval; VLF, very low frequency; LF, low frequency; HF, high frequency. A $P$ value of $<0.05$ is considered statistically significant. ${ }^{\star}$ Significant differences compared with fully recovered patients. ${ }^{*}$ Significant differences compared with uninfected patients.

capacity of the virus to alter the ANS is the so-called "silent hypoxia", a characteristic sign of COVID-19. This condition has been associated with endothelial damage affecting the central and peripheral nervous receptors, altering respiratory control and dyspnea perception. Specifically, the injury has been postulated to occur in the vagal fibers, the glossopharyngeal afferents, and in the nucleus of the tractus solitarius, which are all key in respiratory and autonomic homeostasi ${ }^{23,24}$. In our study, most of the patients could not be evaluated for silent hypoxemia because arterial blood gases were not performed during the acute phase. Nevertheless, nearly all patients with silent hypoxemia are hospitalized at some point, as this condition leads to a critical diagnostic delay; in contrast to our study population of patients with mild disease who did not require hospital admission (therefore, assuming the absence of silent hypoxemia).

Finally, interference of angiotensin II synthesis by COVID-19 can be postulated as the last possible pathophysiological mechanism leading to dysautonomia. As discussed above, SARS-CoV-2 penetrates cells by attaching to the ACE2 receptor, influencing the synthesis of endogenous angiotensin II, a hormone that directly activates the SNS. Dysfunction of the renin-angiotensin-aldosterone system with compensatory activation of the SNS may also contribute to IST. However, our study was unable to demonstrate SNS participation in IST, and further investigations are needed to elucidate and characterize this patho-physiological aspect.

The reasons for the absolute predominance of this pathological phenomenon in young females, the concomitant high prevalence of environmental allergies, and the lack of correspondence with the severity of the index SARS-CoV-2 acute infection remain uncertain. Although IST and POTS are complex, heterogeneous syndromes with overlapping clinical manifestations and potential common mechanisms, it remains important to distinguish between these entities in order to provide the most appropriate treatment. The results of our study suggest that patients with PCS and IST may likely benefit from pharmacological treatment, such as beta-blockers, which blunt the sympathetic nervous system response. However, the pharmacological agent of choice, the timing of its administration, and the clinical response will warrant a separate investigation. 

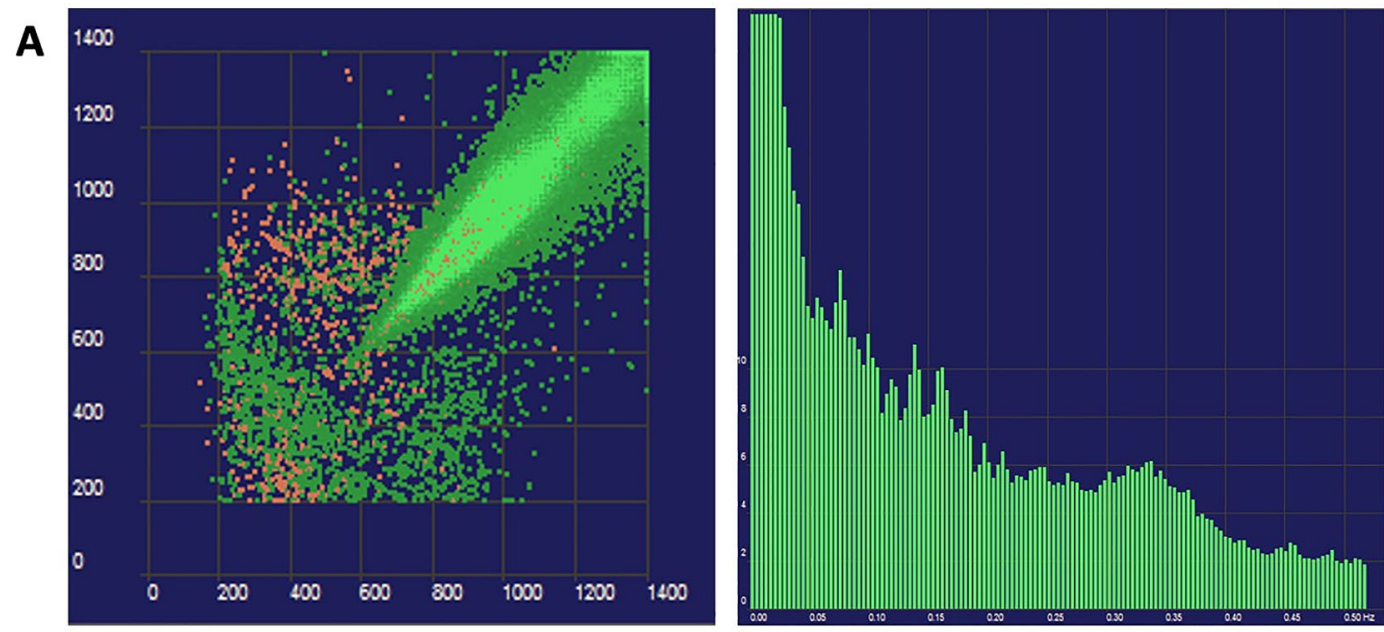

B
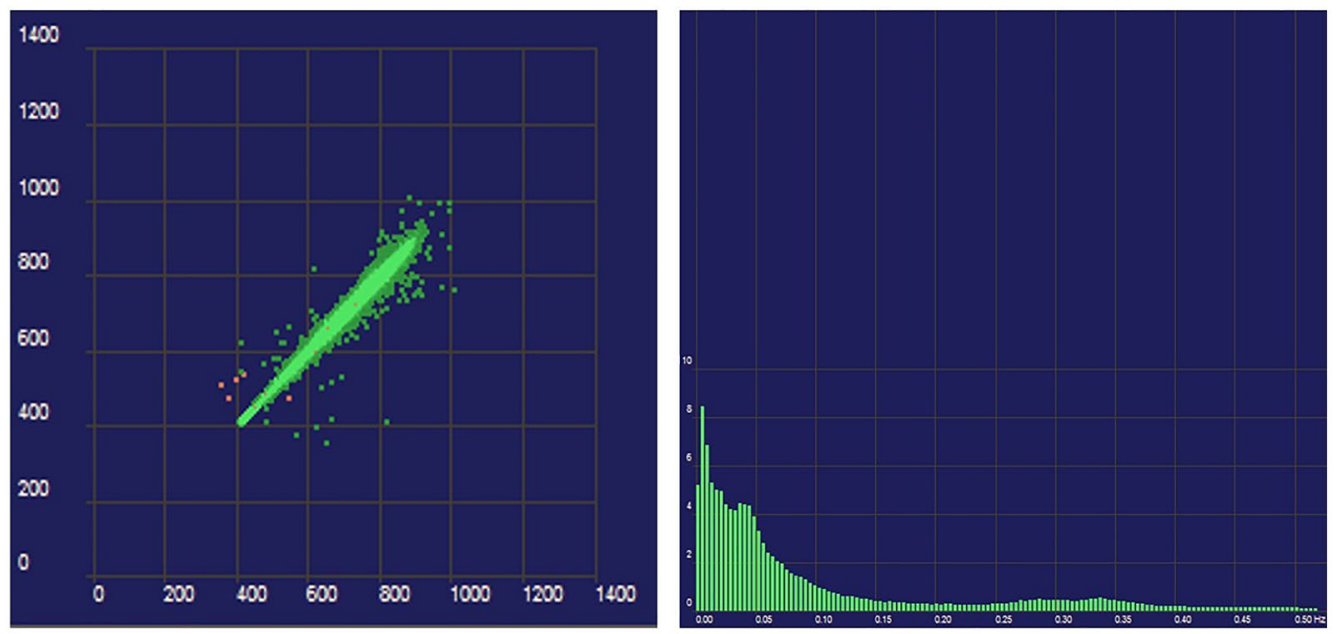

Figure 2. (A) Uninfected subject. Poincaré plot of 24-hour ECG monitoring showing the beat-to-beat variability from an uninfected subject and histogram of the frequencydomain parameters. (B) IST patient. Poincaré plot of 24-h ECG monitoring and histogram of the frequency-domain parameters from a patient with IST. A lower heart rate variability in comparison with the uninfected subject and an overall decrease is observed throughout all bands, being more manifest at the high frequency band (HF, 0.15-0.40 Hz), are both apparent.

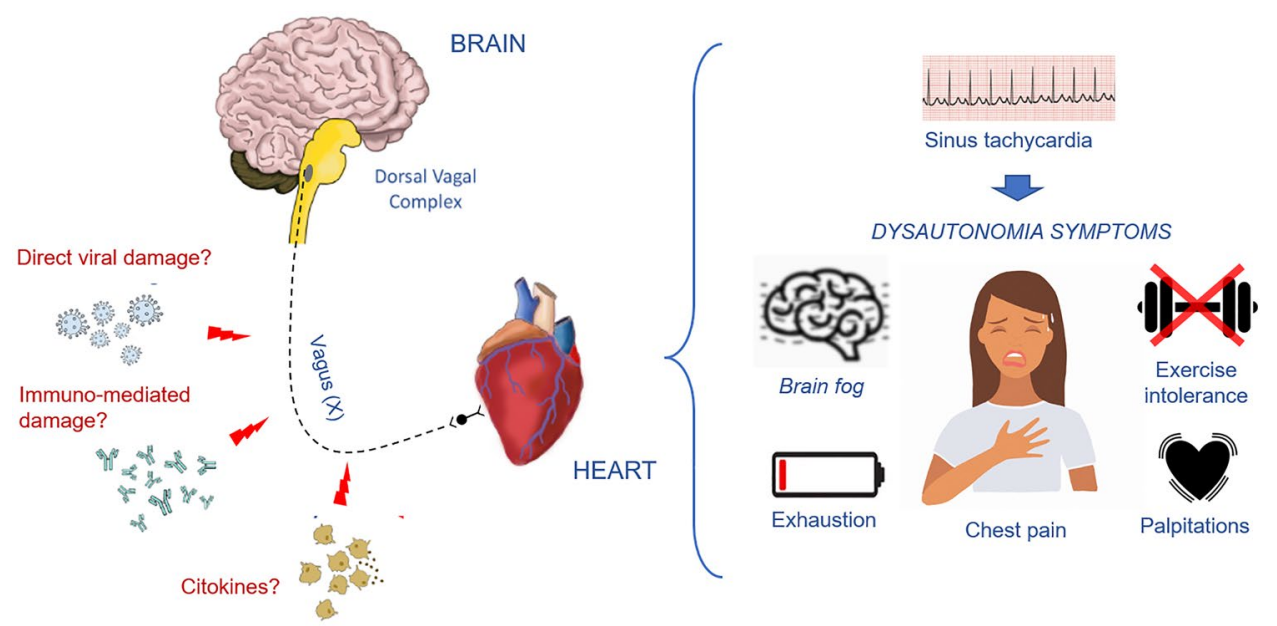

Figure 3. Illustration of the pathophysiological mechanisms underlying Post-COVID-19 syndrome. 


\section{Limitations}

Assessment of ANS function is challenging and barely feasible in daily clinical practice. Standardized reference values extracted from healthy populations are frequently not available. For this reason, we performed the same tests in two gender- and age-controlled groups, one with matched disease stage and severity and one without previous infection. The small size of the control group is also a limitation, and the real incidence of the disease should be ascertained in larger population studies. Despite these limitations, we demonstrated significantly decreased parasympathetic tone among our PCS patient population.

Most of the patients included in this study did not require hospital admission during the acute phase of SARS-CoV-2 infection. Thus, laboratory parameters characterizing a presumable pro-inflammatory state and/ or myocardial damage during the acute infection phase were not available.

\section{Conclusions}

Inappropriate sinus tachycardia is a prevalent condition among PCS patients and should be incorporated as part of the myriad of multi-organ disorders comprising PCS. This disorder may at least partially explain the prevalent symptoms of palpitations, fatigue, and impaired exercise capacity observed in PCS patients. Cardiac ANS imbalance with decreased parasympathetic activity seems to be a plausible pathophysiological explanation for this phenomenon.

Received: 19 June 2021; Accepted: 9 December 2021

Published online: 07 January 2022

\section{References}

1. Carfi, A., Bernabei, R., Landi, F., Gemelli Against COVID-19 Post-Acute Care Study Group. Persistent symptoms in patients after acute COVID-19. JAMA 324(6), 603-605. https://doi.org/10.1001/jama.2020.12603 (2020).

2. COVID-19 rapid guideline: managing the long-term effects of COVID-19. (National Institute for Health and Care Excellence (UK), London, 2020).

3. Dani, M. et al. Autonomic dysfunction in 'long COVID': Rationale, physiology and management strategies. Clin. Med. (Lond.). 21(1), e63-e67. https://doi.org/10.7861/clinmed.2020-0896 (2021).

4. Feigofsky, S. \& Fedorowski, A. Defining cardiac dysautonomia-Different types, overlap syndromes; case-based presentations. J. Atr. Fibrillation. 13(1), 2403. https://doi.org/10.4022/JAFIB.2403 (2020).

5. Goldstein, D. S. The possible association between COVID-19 and postural tachycardia syndrome. Heart Rhythm S1547-5271(20), 31141-31143. https://doi.org/10.1016/j.hrthm.2020.12.007 (2020).

6. Nwazue, V. C. et al. Postural tachycardia syndrome and inappropriate sinus tachycardia: Role of autonomic modulation and sinus node automaticity. J. Am. Heart Assoc. 3(2), e000700. https://doi.org/10.1161/JAHA.113.000700 (2014).

7. Swai, J., Hu, Z., Zhao, X., Rugambwa, T. \& Ming, G. Heart rate and heart rate variability comparison between postural orthostatic tachycardia syndrome versus healthy participants; A systematic review and meta-analysis. BMC Cardiovasc. Disord. 19(1), 320. https://doi.org/10.1186/s12872-019-01298-y (2019).

8. Olshanky, B. \& Sullivan, R. M. Inappropriate sinus tachycardia. J. Am. Coll. Cardiol. 63(8), 793-801. https://doi.org/10.1016/j.jac. 2012.07.074 (2013).

9. Yu, C. M. et al. Cardiovascular complications of severe acute respiratory syndrome. Postgrad. Med. J. 82(964), 140-144. https:// doi.org/10.1136/pgmj.2005.037515 (2006).

10. Madjid, M. et al. Potential effects of coronaviruses on the cardiovascular system: A review. JAMA Cardiol. 5(7), 831-840. https:// doi.org/10.1001/jamacardio.2020.1286 (2020).

11. Goldberger, J. J. et al. Autonomic nervous system dysfunction: JACC focus seminar. J. Am. Coll. Cardiol. 73(10), 1189-1206. https:// doi.org/10.1016/j.jacc.2018.12.064 (2019).

12. Chow, D. et al. Symptoms of autonomic dysfunction in human immunodeficiency virus. Open Forum Infect. Dis. 2(3), ofv103. https://doi.org/10.1093/ofid/ofv103 (2015).

13. Carod-Artal, F. J. Infectious diseases causing autonomic dysfunction. Clin. Auton. Res. 28(1), 67-81. https://doi.org/10.1007/ s10286-017-0452-4 (2018).

14. Mittal, C. M., Wig, N., Mishra, S. \& Deepak, K. K. Heart rate variability in human immunodeficiency virus-positive individuals. Int. J. Cardiol. 94(1), 1-6. https://doi.org/10.1016/j.ijcard.2003.02.002 (2004).

15. Hemachudha, T. et al. Human rabies: Neuropathogenesis, diagnosis, and management. Lancet Neurol. 12(5), 498-513. https://doi. org/10.1016/S1474-4422(13)70038-3 (2013).

16. Abboud, H. et al. COVID-19 and SARS-Cov-2 infection: Pathophysiology and clinical effects on the nervous system. World Neurosurg. https://doi.org/10.1016/j.wneu.2020.05.193 (2020).

17. Low, P. A. \& Sandroni, P. Postural tachycardia syndrome (POTS). Primer Auton. Nervous Syst. https://doi.org/10.1016/B978-0-12386525-0.00106-2 (2012).

18. Schondorf, R. \& Low, P. A. Idiopathic postural orthostatic tachycardia syndrome: An attenuated form of acute pandysautonomia?. Neurology 43(1), 132-137. https://doi.org/10.1212/wnl.43.1_part_1.132 (1993).

19. Zubair, A. S. et al. Neuropathogenesis and neurologic manifestations of the coronaviruses in the age of coronavirus disease 2019: A review. JAMA Neurol. 77(8), 1018-1027. https://doi.org/10.1001/jamaneurol.2020.2065 (2020).

20. Yachou, Y., El Idrissi, A., Belapasov, V. \& Ait, B. S. Neuroinvasion, neurotropic, and neuroinflammatory events of SARS-CoV-2: Understanding the neurological manifestations in COVID-19 patients. Neurol. Sci. 41(10), 2657-2669. https://doi.org/10.1007/ s10072-020-04575-3 (2020).

21. Song, E. et al. Neuroinvasion of SARS-CoV-2 in human and mouse brain. J. Exp. Med. 218(3), e20202135. https://doi.org/10.1084/ jem.20202135 (2021).

22. Karuppan, M. K. M. et al. SARS-CoV-2 infection in the central and peripheral nervous system-associated morbidities and their potential mechanism. Mol. Neurobiol. 58(6), 2465-2480. https://doi.org/10.1007/s12035-020-02245-1 (2021).

23. Rahman, A. et al. Silent hypoxia in COVID-19: Pathomechanism and possible management strategy. Mol. Biol. Rep. https://doi. org/10.1007/s11033-021-06358-1 (2021).

24. Barizien, N. et al. Clinical characterization of dysautonomia in long COVID-19 patients. Sci. Rep. https://doi.org/10.1038/s41598021-93546-5 (2021). 


\title{
Acknowledgements
}

We thank Laia Valls for her collaboration in data collection and Carolina Galvez and Carolina Jaillier for the illustration.

\section{Author contributions}

J.A., V.B. and R.V. wrote the main manuscript text and prepared figures. All authores reviewed the mansucript.

\section{Funding}

This study did not receive any specific funding.

\section{Competing interests}

The authors declare no competing interests.

\section{Additional information}

Correspondence and requests for materials should be addressed to R.V.

Reprints and permissions information is available at www.nature.com/reprints.

Publisher's note Springer Nature remains neutral with regard to jurisdictional claims in published maps and institutional affiliations.

\begin{abstract}
(c) (i) Open Access This article is licensed under a Creative Commons Attribution 4.0 International License, which permits use, sharing, adaptation, distribution and reproduction in any medium or format, as long as you give appropriate credit to the original author(s) and the source, provide a link to the Creative Commons licence, and indicate if changes were made. The images or other third party material in this article are included in the article's Creative Commons licence, unless indicated otherwise in a credit line to the material. If material is not included in the article's Creative Commons licence and your intended use is not permitted by statutory regulation or exceeds the permitted use, you will need to obtain permission directly from the copyright holder. To view a copy of this licence, visit http://creativecommons.org/licenses/by/4.0/.
\end{abstract}

(c) The Author(s) 2022 